\title{
Interoperability in Connected Vehicles - A Review
}

\author{
Shaik Mazhar Hussain ${ }^{\mathrm{a}}$, Kamaludin Mohamad Yusof ${ }^{\mathrm{b}}$, Shaik Ashfaq Hussain ${ }^{\mathrm{c}}$ \\ ${ }^{\mathrm{a}, \mathrm{b}, \mathrm{c}}$ School of Electrical Engineering (SEE), Department of Communications Engineering and Advanced \\ Telecommunication Technology (ATT), Faculty of Engineering Universiti Teknologi Malaysia, Malaysia, Johor \\ Bahru
}

Received: 12 May 2019; Accepted: 09 August 2019; Published: 08 September 2019

\begin{abstract}
The introduction of automated transportation system and implementation of connected vehicle system has made interoperability even more critical than before in order to ensure effective connectivity, consistency , accuracy and reliable performance.Connected vehicles are employed with in vehicle sensor networks to collect , store, process and communicate with neighbouring vehicles to help drivers making life saving decisions. Connected vehicle systems enable the exchange of communication between a vehicle and world. Some vehicles may only receive communication, other vehicles may only send data and some may receive and send data. Hence it is essential that all systems, devices need to work together in a safe, trusted, efficient manner. Devices/Sensors embedded in vehicles follow different communication protocols as the sensors or devices are manufactured by different vendors. Hence management of coordination between devices is of much importance in Dynamic, Mobile, Heterogeneous environments such as connected vehicles. Hence, there is a need to create seamless interoperability to enable connected vehicles. It is required to enable intelligent transportation system (ITS) system/devices in Vehicles to effectively connect and communicate with other systems/devices. The adaptability of Interoperability when the devices are adhere to dynamics and mobile in nature becomes tedious and cumbersome. Solutions to interoperability is not much explored in literature. Existing interoperability solutions does not guarantee to articulate for high mobile environments such as connected vehicles where vehicles are highly mobile in nature and moving at varying speeds. The paper summarizes connected vehicles issues and challenges, current interoperability solutions, the impact of interoperability in connected vehicle scenario and demonstrates the effect of conversion time delay when exchanging data between source and destination pairs.
\end{abstract}

Index Terms: Connected Vehicle, Interoperability, V2V Communication, DSRC/WAVE, High mobile environments, varying speeds

(C) 2019 Published by MECS Publisher. Selection and/or peer review under responsibility of the Research Association of Modern Education and Computer Science

\footnotetext{
* Corresponding author.

E-mail address: hussain@graduate.utm.my,kamalmy@utm.my,sk.ashfaq.hussain@gmail.com
} 


\section{Introduction}

A connected vehicle is equipped with internet access usually local area networks(LAN). This allows the vehicles to share internet inside and outside the vehicle. Vehicles communicate through a wireless communication protocol called Dedicated Short Range Communications (DSRC) operating in 5.9GHz band with a latency of 0.02 seconds .It is predicted by 2022, there would be more than 125 million vehicles connected. During the development stages of connected vehicles, the primary purpose was safety and emergency for a vehicle whenever there is an accident. Advancements in transportation has brought different ways to communicate and connect with vehicles and its surroundings [1,2]. Table 1 shows the types of vehicle connectivity The main scope of this paper is limited to Vehicle to Vehicle communication(V2V). V2V communication enables vehicles to wirelessly exchange their information about location and speed. V2V communication follows broadcasting of messages and receives omni directional messages as fast as 10 times per second creating $360^{\circ}$ awareness of other vehicles in surroundings. Vehicles are equipped with appropriate softwares to process the messages received from neighbouring vehicles and to determine any crashes, traffic congestion and hence ensures safety environment. Some of the connected vehicle applications are intersection movement assist, donot pass warning, emergency electronic brake light application, blind spot warning .The hardware in connected vehicles can be divided into two types. One is built in and the other is brought in connection systems. Built in connected systems have a internet connections via GSM where as brought in connections are plugged into on-board diagnostics port for accessing vehicle data. Brought in devices are divided in to two types of connection.

1. For the internet connection, hardware relies on users smart phone.However, smart phone is actually a kind of hardware

2. Hardware establishes internet connection via GSM module.

Table 1. Vehicle technologies, connectivity and communication $[3,4,5]$

\begin{tabular}{|l|l|l|}
\hline TYPES OF CONNECTIVITY & OPERATION & TYPE OF COMMUNICATION \\
\hline Vehicle to Infrastructure & $\begin{array}{l}\text { This technology Provides information } \\
\text { about the infrastructure to the } \\
\text { driver/user }\end{array}$ & $\begin{array}{l}\text { Safety, mobility, environment related } \\
\text { conditions }\end{array}$ \\
\hline Vehicle to Vehicle & $\begin{array}{l}\text { This technology Speed, position of } \\
\text { neighbouring vehicles through wireless } \\
\text { technology }\end{array}$ & $\begin{array}{l}\text { Avoid accidents , ease traffic } \\
\text { congestions }\end{array}$ \\
\hline Vehicle to Cloud & $\begin{array}{l}\text { This technology Vehicles exchange the } \\
\text { information with the cloud. Uses the } \\
\text { information from other vehicles }\end{array}$ & $\begin{array}{l}\text { As cloud is connected with industries , } \\
\text { transportation energy and smart } \\
\text { homes. Vehicles make use of it }\end{array}$ \\
\hline Vehicle to Pedestrian & $\begin{array}{l}\text { This technology senses information } \\
\text { about environments }\end{array}$ & $\begin{array}{l}\text { Communicates to surrounding vehicles , } \\
\text { infrastructure and personnel mobile } \\
\text { devices. }\end{array}$ \\
\hline
\end{tabular}

DSRC/WAVE is an emerging wireless standards for wireless mobile communication system that supports vehicle to vehicle (V2V) and vehicle to infrastructure (V2I) communications for Intelligent Transportation systems(ITS). Some of the features of DSRC/WAVE are high data rates, low latency and high mobility communications. Table. 2 shows the comparision of DSRC/WAVE to various wireless systems.Besides benefits, Some of the drawbacks and challeneges faced by connected vehicles are Hackability as and when vehicles connected to the internet are more exposed to the outside world, Reliability as cars, sensors and networks will malfunction, Privacy as the data gathered should not be used for unauthorised purposes. One of 
the major goals of connected vehicles is to enable seamless interoperability among consumers, devices, vehicles and various computational platform [6]. Lack of standardization, data silos, heterogeneity among devices are the major issues needs to be addressed. Achieving Semantic interoperability in connected vehicles is cumbersome and plays a vital role when it comes to information exchange since the systems used in the vehicles are developed by different organizations and follow different formats. Interoperability are classified into three types 1) Syntactic interoperability 2) Semantic Interoperability and 3) Cross domain Interoperability. Semantic Interoperability puts a lot of concerns about connected vehicles. For the vehicles to exchange the information unambiguously, there is a need that the systems should agree on the meaning of the data that they are exchanging. Hence the Connected vehicles must ensure semantic interoperability. However, failure in facilitating semantic interoperability between heterogeneous information systems could lead to disastrous results [7]. Several approaches have been proposed to solve interoperability problem. Solutions have been developed for static environments $[8,9,10,11,12,13,14,15]$. However, there is a need to develop solutions and techniques in dynamic and highly mobile environments where the device characteristics are not the same all the time. The central idea of "connected vehicles" is the vehicles should interoperate semantically without any human intervention and persists collaboration between vehicles. Hence to sustain in a long term, bridging semantic interoperability is of utmost importance. Semantic technologies substantially provides a solution to semantic interoperability by integrating vehicles to web. However, Mapping between the systems still remains an indispensable problem to be considered. Existing solution to interoperability $[16,17]$ proposed "model driven approach", where a concept of mapping method is used to measure the effect of data elements on model transformations. Results have been demonstrated where information transfer is measured. The authors have emphasized on measuring Interoperability capacity by characterizing information transfer through representing Model transformations. However, Increase in data elements consequently leads to more execution time. Information recoverability is one of the major challenge that needs to be considered while developing semantic interoperability in a long term. Connected vehicles is a different terminology but it is still a part of IOT where vehicles are treated as IOT devices [18]. Connected vehicles are housed with sensors that collects the information from surroundings requires complex computations, scalability, sustainability and extensibility [19]. Vehicles need to extract the information about its surroundings and able to communicate with other vehicles with in a short delay. Information about various road, traffic and vehicle conditions should be locally processed and exchanged between other vehicles. Information collected from the ' $n$ ' vehicles follows different formats, different data types which requires efficient data processing techniques locally and globally to deal with. All these concerns put together to urge in building an architecture for semantic interoperability and the layers that could integrate vehicles for information exchange. A detailed comprehensive survey is made on architectures, protocols and enabling technologies in IOV [20]. In one of the literature paper [21,22,23], the authors have explored several existing and proposed approaches using Middle Ware for interoperability. Middle ware solutions are well supported for network interoperability. However, lacks Syntactic and Semantic Interoperability. Middle ware approaches plays a vital role when information from one network is exchange between other networks. In addition, one of the major concern is with the Wi-Fi AP Connection time which will be very few seconds that could deliberately limits the amount of data transferred in one connection. This could lead to a major challenge and raises a big question "How well the data can be exchanged and interpreted between the vehicles in such extremely less time where latency plays a major role in the context of connected vehicles". All these issues and concerns put together evolves research questions which needs to be addressed and will be answered in the research proposal.

i. What are the semantic interoperability requirements for connected vehicles?

ii. To meet the semantic requirements of connected vehicles, can a framework be developed?

iii. How does the performance of connected vehicles be evaluated in terms of Semantic Interoperability?

This paper focuses mainly on current issues and challenges of connected vehicles and highlights one of the major issue and challenege of connected vehicle i.e Interoperability and discusses the existing solutions of 
interoperability. Finally the paper demonstartes the effect of conversion time during data exchange between source and destination and how it impacts when considered in connected vehicle scenarios.

Below table shows comparision of DSRC/WAVE to various wireless systems

Table 2. Comparision of DSRC/WAVE to various wireless systems [32]

\begin{tabular}{|c|c|c|c|c|}
\hline & DSRC/WAVE & Wi-Fi & Cellular & \begin{tabular}{|l} 
Mobile \\
WiMAX5
\end{tabular} \\
\hline Data rate & 3-27Mbps & 6-54Mbps & $<2 \mathrm{Mbps}$ & $1-32 \mathrm{Mbps}$ \\
\hline Latency & $50 \mathrm{~ms}$ & Seconds & Seconds & $?$ \\
\hline Range & $<1 \mathrm{~km}$ & $<100 \mathrm{~m}$ & $<10 \mathrm{~km}$ & $<15 \mathrm{~km}$ \\
\hline Mobility & $60 \mathrm{mph}$ & $<5 \mathrm{mph}$ & $>60 \mathrm{mph}$ & $80 \mathrm{mph}$ \\
\hline $\begin{array}{l}\text { Nominal } \\
\text { Bandwidth }\end{array}$ & $10 \mathrm{MHz}$ & $20 \mathrm{MHz}$ & $<3 \mathrm{MHz}$ & $<10 \mathrm{MHz}$ \\
\hline $\begin{array}{l}\text { Operating } \\
\text { Band }\end{array}$ & $\begin{array}{l}5.86-5.92 \mathrm{GHz} \\
\text { (ITS-RS) }\end{array}$ & $\begin{array}{l}2.4 \mathrm{GHz} \\
5.2 \mathrm{GHz} \\
(\mathrm{ISM})\end{array}$ & $\begin{array}{l}800 \mathrm{MHz} \\
1.9 \mathrm{GHz}\end{array}$ & $2.5 \mathrm{GHz}$ \\
\hline IEEE std. & $\begin{array}{l}802.11 \mathrm{p} \\
\text { (WAVE) }\end{array}$ & $802.11 \mathrm{a}$ & $\mathrm{N} / \mathrm{A}$ & $802.16 \mathrm{e}$ \\
\hline
\end{tabular}

\section{Literature Review}

A comprehensive review on the existing works related to Interoperability is thoroughly studied and detailed conclusions were drawn. In [24], plug ' $n$ ' interoperate approach is used . The steps needed to perform the information exchange between devices are described by interoperability specification. More than one IS can exist to describe the information exchange between each pair of devices. There is the need to measure the information exchange described by each one. The limitation of this paper is "Execution time will start to increase as more number of data elements are increased". In [25] the research paper is mainly focused on proposing solution to interoperability in smart homes because of heterogeneity of devices. Web services has been proposed as solution to solve interoperability in Home environmnets. Simple Access Object protocol (SOAP) technology is used to solve interoperability issues. The solution is implemented for static environments. In [26], the authors have highlighted the issue of interoperability in connected vehicles. The authors have suggested a solution using web services (a cloud platform).A standard protocol will be developed using XML. The vehicles communicate with other vehicles through web. The protocols will be translated to a standardized protocol and communication takes place. The time needed to process the data could lead to Latency issues. In [27], the authors present a hub-based approach for IoT Interoperability. This Hub includes various data conversion features. However it is not about dynamic solution. In [28] the authors have proposed cloud based platform as a solution to provide interoperability. However, the collected data need to be stored locally and analyzed. The availability of local storage is important to avoid the unpredictable latencies from wireless transmission of data to the cloud. In [29] , Evolution of IOV is discussed. Research and Engineering challenges are identified. Some of the challenges include coexistence of cloud, edge computing, data caching strategies at the edge Integration of data processing and management of IOV services Seamless Interoperability among vehicular sensors IOT architecture is presented. The authors have considered vehicles as IOT resources and provides mechanisms to integrate them in an IOV eco system. Also seamless interoperation among components such as sensors, computational platform and consumers. The functional elements and operational stages of the architecture also assist in maintaining interoperability among the components. The authors in this paper is mainly focusing on Computational platforms such as cloud and Edge computing to access the data in a very short period. In addition, the authors have mentioned for information to flow and correctly interpreted, a 
unified IOT and ITS architecture is required. In [30], Traditional methods of interoperability including protocol gateways and adapters are often used at network layer. Middle ware and protocol proxy is used for application interoperability. However it drags the problem of interoperability due to scalability issues, reconfiguration effort, processing overheads. The paper has proposed a secure, on demand transparent protocol translator for the IIOT. The paper is mainly focused on Interoperability in communication protocols. The proposed protocol is not a middle ware and based on service oriented architecture based participant. The proposed idea is basically on demand when needed. Operates transparently, supports low latency and secured using arrow head authorization and authentication. Early work on Interoperability in communication protocol is concentrated on network layer. Interoperability in application layer is not addressed much.

\section{Research Design and Analysis}

This section discusses results obtained from one of the existing papers titled "Measuring data transfer in heterogeneous IOT environments "[31] and demonstrates the effect of conversion time as data elements increases. In the paper [31], The authors have addressed the problems of heterogeneity in devices which leads to information exchange problem. The approach adapted by the authors is "Plug $n$ Interoperate" approach where interoperability specifications (IS specifications) are described. The authors of the paper have clearly mentioned that there could be a possibility of more than one IS to exist for information exchange. To select suitable IS between pair of devices, there is a need to measure information exchange described by each one. Several methods are existing in literature but they totally rely on data formats and deep understanding of IS, In order to overcome this, advance measurement methods are proposed by authors. One such method is Plug and Interoperate approach. In this method, measurement of information exchange is done without deep understanding of IS. One of the major issues that is identified with this approach is time delay problem that is the time needed to process the data before communicating with other devices. To showcase this problem, HASH algorithm is used and the paper is simulated using MATLAB considering the same parameters such as data transfer ratio and conversion time is calculated with respect to data transfer ratio. Two different codes were written Data transfer ratio and conversion time. From Figure 6, It is clear that the conversion time increases as the data transfer ratio increases.

Data transfer ratio: number of elements defined in source and target models and number of elements used in element mapping to perform the information exchange represented in the source and target data. To compute data transfer ratio, there are four parameters considered

Source Model Count (SMC) : Number of data elements defined in the source model

Source Data Count (SDC): Number of data elements in source data used by element mapping

Target Model Count (TMC) : Number of data elements defined in the target model

Target Data Count (TDC) : Number of data elements in the target data that are produced by execution of element mapping. Figure 1 to Figure 6 illustrates the Simulation results. 


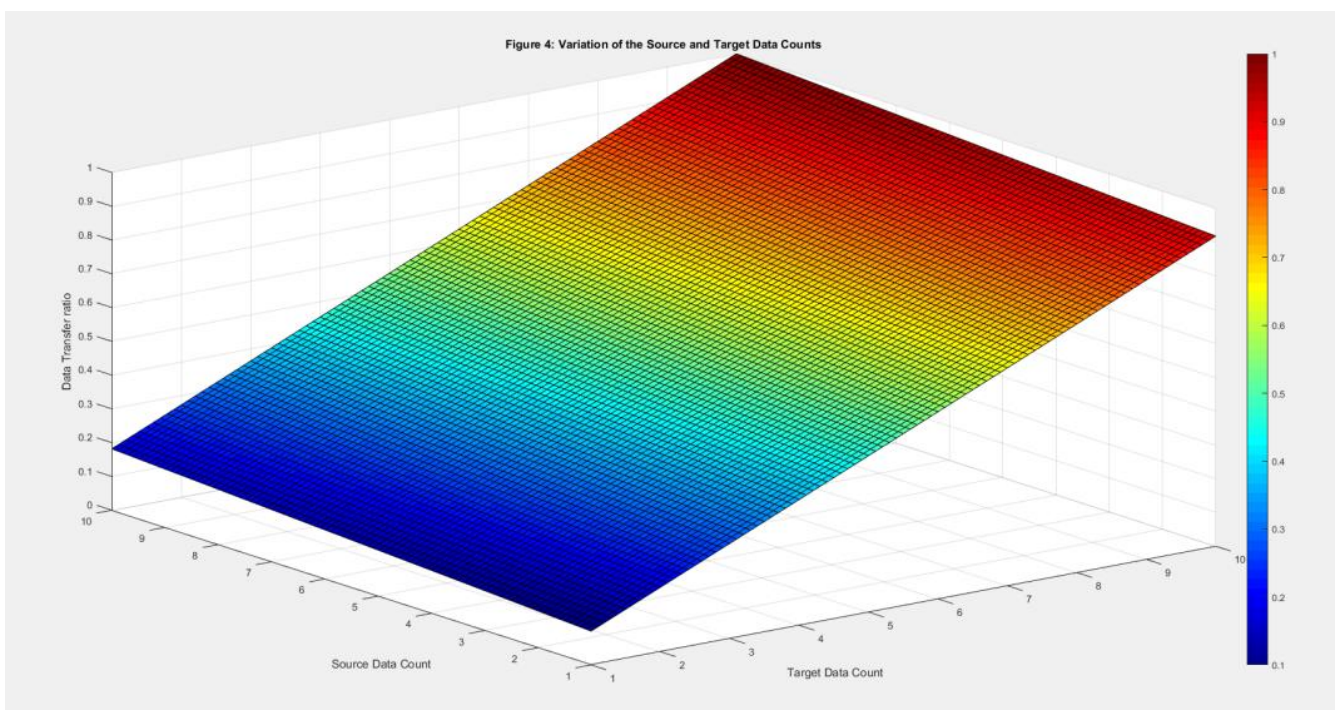

Fig.1 Variation of the source and target data counts [31]

Increase of either the Source Data Count or the Target Data Count results in the increase of the Data Transfer ratio

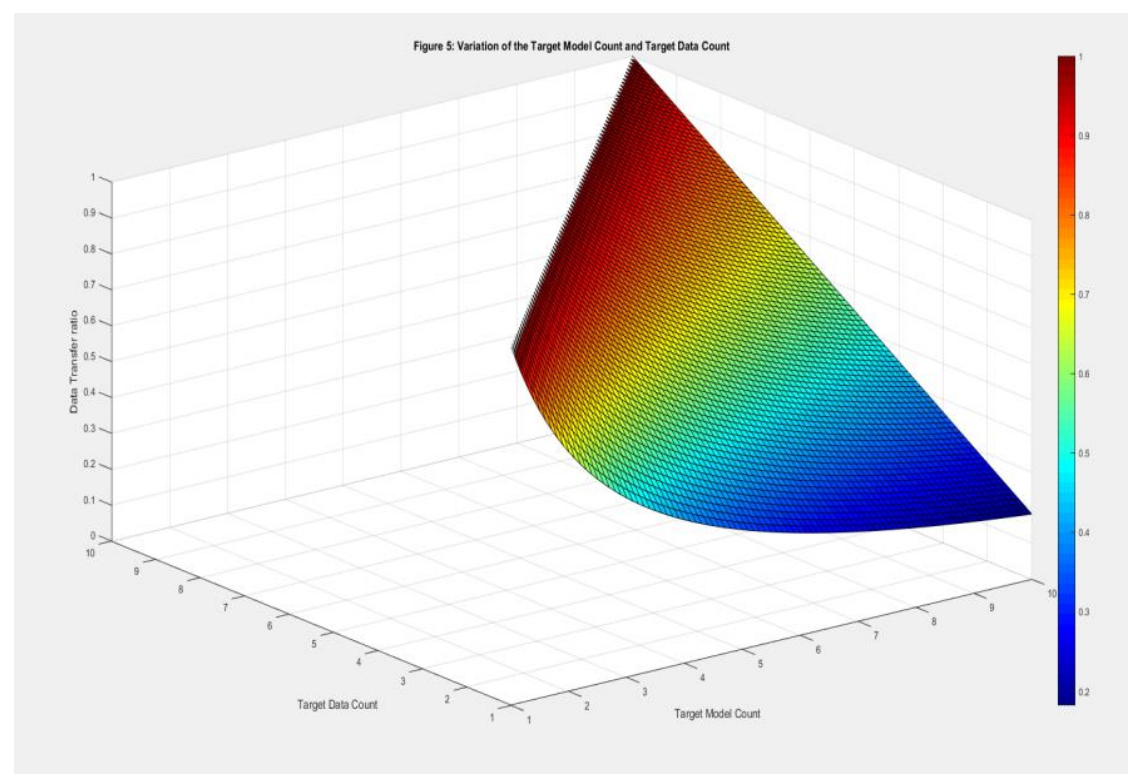

Fig. 2 Variation of the target model count and target data count [31]

When the Target Data Count and the Target Model Count are equal, the Data Transfer ratio reaches its maximum. 


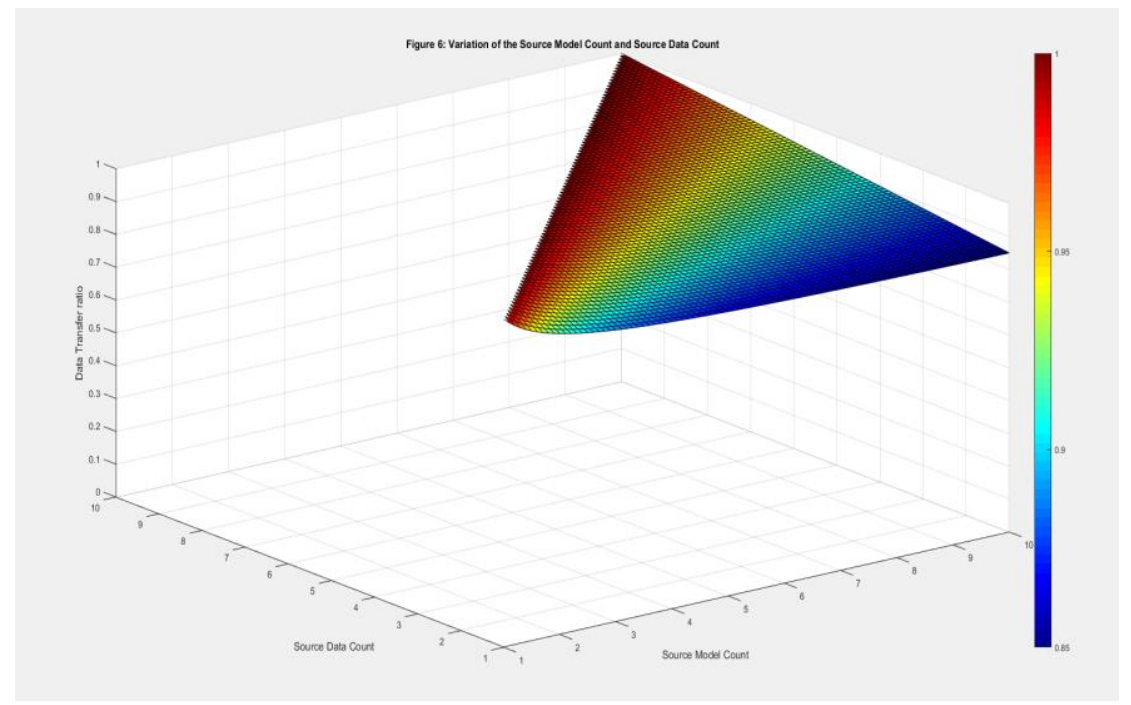

Fig.3 Variation of the source model count and source data count [31]

When the Source Model Count is equal to the Source Data Count, Data Transfer ratio hits the scale maximum.

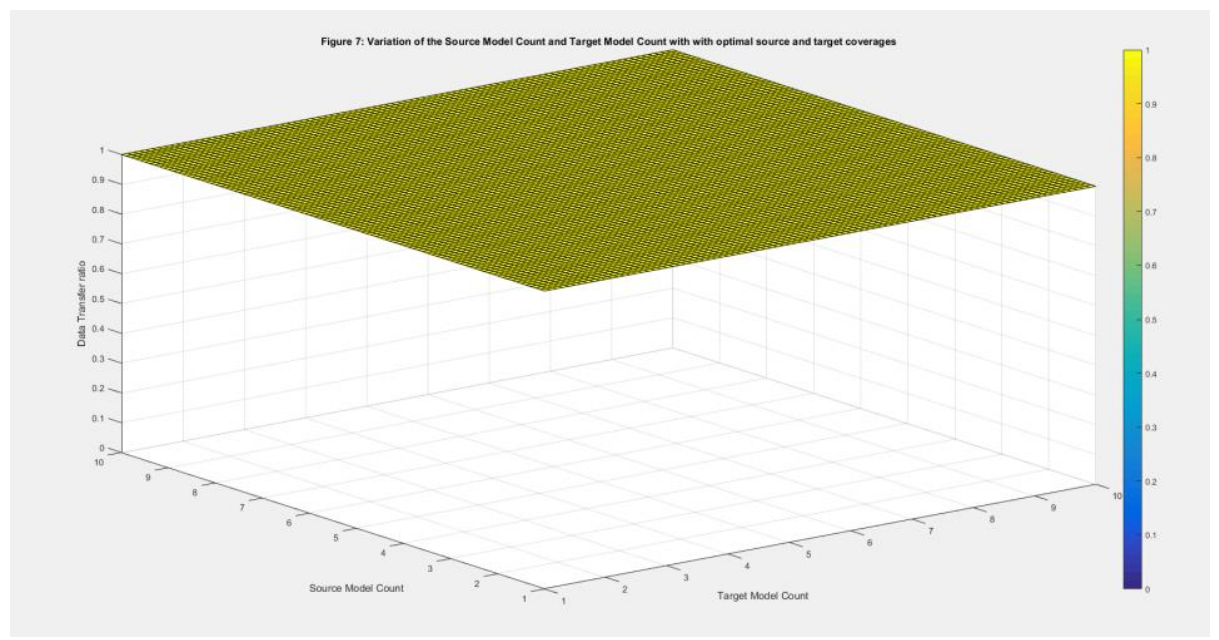

Fig.4. Variation of the source model count and target model count with optimal source and target coverages [31]

This test represents the scenario where exist several interoperability specifications between disparate models, describing each interoperability specification the optimal data exchange. As the ratios between the target counts and the source counts is 1 , then is expected that the Data Transfer Ratio will always have the maximum value. Through the analysis of the obtained graph, it is verified that for any combination of source and target models, respectively represented by Source Model Count and the Target Model Count, the Data Transfer ratio is optimal.

From the below figure, Source model is produced by generating random data .Secure Hash Algorithm (SHA-256) is used as a transformation script to produce target models. 


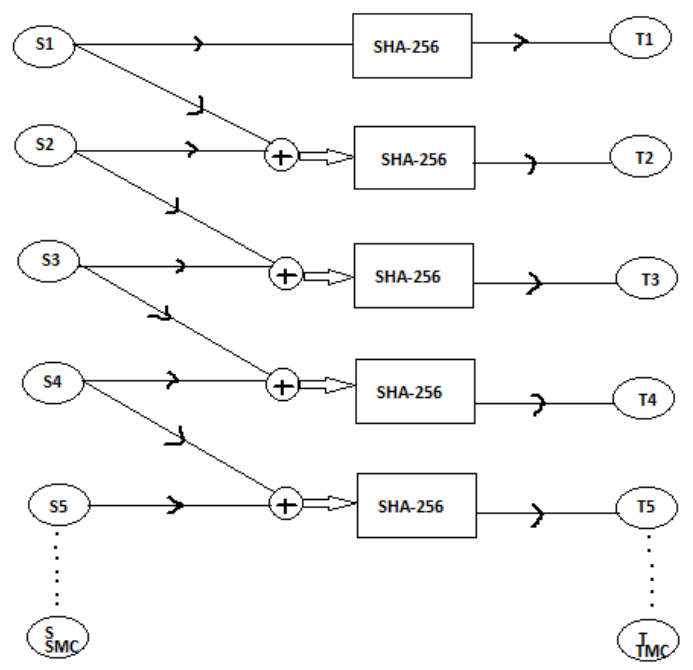

Fig.5. Conversion of source model to target model using SHA-256

Fig.6. Data Transfer Ratio vs Conversion Time

Finally the above figure shows that as the data transfer ratio increases there is an increase in conversion time which is linear in nature. Hence this type of approaches are not suitable when dealing with mobile devices and where the time delay plays a crucial role. If the conversion time delays are not maintained properly in a mobile environments such as when exchanging data between vehicles, this could end up with disastrous results. Hence optimized mechanisms should be developed to ensure high conversion accuracy and low latency requirements $\sim 0.02$ seconds.

\section{Conclusions}

In our proposed work, Conversion time is computed during information exchange between source and destination. It is clear that as the data transfer ratio increases, the conversion time is drastically increasing which could lead to ambiguity in connected vehicle scenarios where vehicles are highly mobile and dynamic in 
nature. The results signifies the importance of interoperability in connected vehicles. Based on the results obtained, the conversion time, accuracy of conversion and latency are the crucial parameters that needs to be focused when considering interoperability in connected vehicles.

\section{References}

[1] M. Turner, "The future of the connected car," MASHABLE, 26 feb 2011. [Online]. Available: https://mashable.com/2011/02/26/connected-car/. [Accessed 10 may 2019].

[2] Meola, "Automotive Industry Trends: IoT Connected Smart Cars \& Vehicles," Business Insider, 20 dec 2016. [Online]. Available: https://www.businessinsider.com/internet-of-things-connected-smart-cars2016-10?r=UK. [Accessed 1005 2019].

[3] "connected and automated vehicles," Centre for advanced automotive technology, [Online]. Available: http://autocaat.org/Technologies/Automated_and_Connected_Vehicles/. [Accessed 510 2019].

[4] "Intelligent Transportation Systems Joint Program Office," United states department of transportation, [Online]. Available: https://www.its.dot.gov/v2i/. [Accessed 1005 2019].

[5] "Vehicle-to-Vehicle Communication," NHTSA, [Online]. Available: https://www.nhtsa.gov/technologyinnovation/vehicle-vehicle-communication. [Accessed 105 2019].

[6] W. S. E. C. N. N. X. S. R. N. C. a. A. V. V. A. Vinel, "“Enabling wireless communication and networking technologies for the Internet of Things [guest editorial],"," Wireless Commun.,, vol. 23, no. 5, pp. 8-9, 2016.

[7] M. S. a. S. Madnick., "A metadata approach to resolving semantic conflicts.," in In Proceeding of the 17th International Conference on Very Large Data Bases, Spain, 1991.

[8] J. P. S. M. O. U. A. A. E. M. a. C. P. F. Vega, " "An IoT-based open platform for monitoring non-ionizing radiation levels in Colombia,"," in IEEE Colombian Conference on Communications and Computing (COLCOM), , Cartagena, Colombia, 2016.

[9] R. R. C. Y. L. S. M. a. K. S. T. Perumal, "" Interoperability Among Heterogeneous Systems in Smart Home Environment,"," in IEEE International Conference on Signal Image Technology and Internet Based Systems, Bali,Indonesia, 2008.

[10] I.-Y.-Z.-H. P.Agrawalc, ""Choices for interaction with things on Internet and underlying issues.,"," ELSEVIER, vol. 28, pp. 68-90, 2015.

[11] C.-E. L. a. K.-D. Moon, ""Design of a universal middleware bridge for device interoperability in heterogeneous home network middleware.,"," in IEEE, Las Vegas, NV, USA, 2005.

[12] P. S. a. S. R. Sarangi, " "Internet of Things: Architectures, Protocols, and Applications," in " in Journal of Electrical and Computer Engineering", Delhi, 2017.

[13] H.-M. J. J.-Y. O. a. S.-J. K. M.-J. Lee, " "Multimedia room bridge adapter for seamless interoperability between heterogeneous home network devices,"," in IEEE, Las Vegas , NV , USA, 2006.

[14] E. P. a. A. Acquaviva, "'IoT platform for Smart Cities: Requirements and implementation case studies,"," in IEEE 2nd International Forum on Research and Technologies for Society and Industry Leveraging a better tomorrow (RTSI), , Bologna,Italy, 2016.

[15] J.-H. P. a. N. K. H. Park, ""A framework for Interoperability of Heterogeneous devices in Ubiquitous Home.,"," in Second International Conference on Advances in Future Internet, Venice , Italy, 2010.

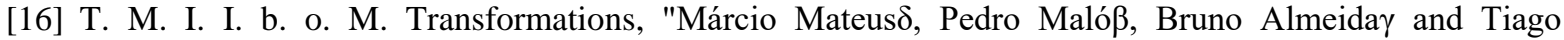
Teixeira, " in 6th Iberian Conference on Information Systems and Technologies (CISTI 2011), Chaves, Portugal , 2011.

[17] T. Teixeira, P. Maló, B. Almeida and M. Mateus, "Towards an Interoperability Management System," in 6th Iberian Conference on Information Systems and Technologies (CISTI 2011), Chaves, Portugal , 2011.

[18] H. S. H. a. G. T. S. Abdelhamid, " "Vehicle as a resource (vaar),"," IEEE Netw.,, vol. 29, no. 1, pp. 12-17, 2015. 
[19] R. P. F. D. C. J. H. C. B. Soumya Kanti Datta, "Integrating Connected Vehicles in Internet of Things Ecosystems: Challenges and Solutions," in 17th International Symposium on a world of wireless,mobile and multimedia networks, coimbra,portugal, 2016.

[20] S. Z. a. J. G.-I. Juan Contreras-Castillo, "Internet of Vehicles: Architecture, Protocols, and Security," IEEE Internet of things Journal, pp. 1-1, 2017.

[21] M. A. Razzaque, M. Milojevic-Jevric and a. S. C. Andrei Palade, "Middleware for Internet of Things: A Survey," IEEE INTERNET OF THINGS JOURNAL, vol. 3, no. 1, pp. 70-95, 2016.

[22] M. G. V. M. S. N. a. Q. Z. S. Anne H. Ngu, "IoT Middleware: A Survey on Issues and Enabling Technologies," IEEE INTERNET OF THINGS JOURNAL, vol. 4, no. 1, pp. 1-20, 2017.

[23] F. a. I. A. O. o. R. Opportunities, "Mung Chiang;Tao Zhang," IEEE INTERNET OF THINGS JOURNAL, vol. 3, no. 6, pp. 854-864, 2016.

[24] P. Maló, M. Mateus, B. Almeida and T. Teixeira, "Measuring Data Transfer in Heterogeneous IoT Environments," in 2013 IEEE International Conference on Green Computing and Communications and IEEE Internet of Things and IEEE Cyber, Physical and Social Computing, Beijing, China, 2013.

[25] T. Perumal, A. R. Ramli, C. Y. Leong, S. Mansor and K. Samsudin, "Interoperability among Heterogeneous Systems in Smart Home Environment," in 2008 IEEE International Conference on Signal Image Technology and Internet Based Systems, Bali, Indonesia, 2008.

[26] Z. Sheng, S. Yang, Y. Yu, A. V. Vasilakos, J. A. Mccann and K. K. Leung, "A survey on the ietf protocol suite for the internet of things: standards, challenges, and opportunities," in IEEE Wireless Communications, 2013.

[27] i. A. h.-b. approach, "Michael Blackstock ; Rodger Lea," in 2014 International Conference on the Internet of Things (IOT), Cambridge, MA, USA, 2014.

[28] S. K. Datta, R. P. F. D. Costa, J. Härri and C. Bonnet, "Integrating connected vehicles in Internet of Things ecosystems: Challenges and solutions," in 2016 IEEE 17th International Symposium on A World of Wireless, Mobile and Multimedia Networks (WoWMoM), Coimbra, Portugal, 2016.

[29] S. K. Datta, J. Haerri, C. Bonnet and R. F. D. Costa, "Vehicles as Connected Resources: Opportunities and Challenges for the Future," in IEEE Vehicular Technology Magazine, 2017.

[30] H. Derhamy, J. Eliasson and J. Delsing, "IoT Interoperability-On-Demand and Low Latency Transparent Multiprotocol Translator," in IEEE Internet of Things Journal, 2017.

[31] M. M. B. A. a. T. T. P. Maló, "Measuring Data Transfer in Heterogeneous IoT Environments," in ," in 2013 IEEE International Conference on Green Computing and Communications and IEEE Internet of Things and IEEE Cyber, Physical and Social Comput.

[32] Y. L. Morgan, "Managing DSRC and WAVE standards operations in a V2V scenario," International Journal of Vehicular Technology, vol. 2010, p. 18, 2010.

\section{Authors' Profiles}

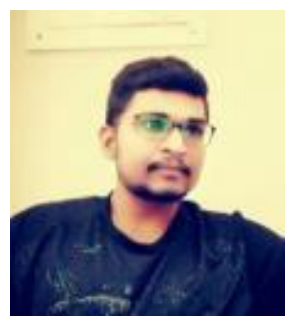

Shaik Mazhar Hussain, Department of Communications Engineering, Advanced Telecommunication Technology (ATT) received his Master's degree in Embedded System from India in 2012 and received PGCert from Coventry University, UK in December 2017 . He is currently a Ph.D candidate at the Universiti Teknology Malaysia (UTM), Johor Bahru, Malaysia. His Research areas of interest include Wireless communications and Intelligent Transportation System (ITS). 


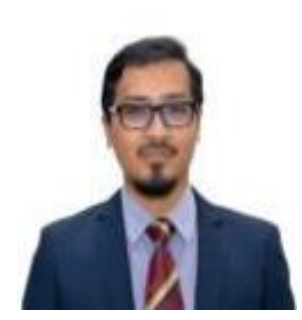

Dr. Kamaludin Mohamad Yusof is a senior lecturer at the School of Electrical Engineering, Universiti Teknologi Malaysia (UTM), Skudai Johor. Graduated with a Bachelor of Electrical Engineering (Electronics) (2000), Master of Electrical Engineering (2003) from UTM and Phd in Electrical engineering University of Essex UK (2014). He has involved in several research areas such as Internet of Thing, Internet Networking and Network Security.

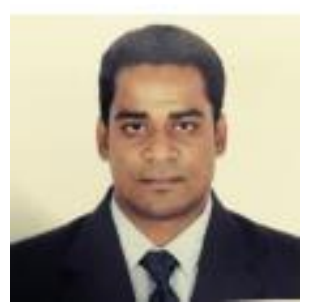

Shaik Ashfaq Hussain, Department of Communications Engineering, Advanced Telecommunication Technology (ATT) received his Master's degree in Embedded and VLSI System design from India in 2013. He is currently a Ph.D candidate at the Universiti Teknology Malaysia (UTM), Johor Bahru, Malaysia. His Research areas of interest include Wireless communications and Intelligent Transportation System (ITS).

How to cite this paper: Shaik Mazhar Hussain, Kamaludin Mohamad Yusof, Shaik Ashfaq Hussain, "Interoperability in Connected Vehicles - A Review", International Journal of Wireless and Microwave Technologies(IJWMT), Vol.9, No.5, pp. 1-11, 2019.DOI: 10.5815/ijwmt.2019.05.01 